\title{
Influence of Formulation Change on Drug Release Kinetics from Hydroxypropylmethylcellulose Matrix Tablets
}

\author{
Guojie $\mathrm{XU}^{a}$ and Hisakazu SunADA* ${ }^{*, b}$ \\ Shenyang Pharmaceutical University, ${ }^{a} 103$ Wenhua road Shenhe district, Shenyang, Liaoning provice, 110015 China, \\ and Faculty of Pharmacy, Meijo University," Yagotoyama, Tempaku-ku, Nagoya 468, Japan. \\ Received August 31, 1994; accepted November 4, 1994
}

\begin{abstract}
Examination was made of the release of indomethacin from hydroxypropylmethylcellulose (HPMC) matrices and the results were found to usually follow first order release kinetics. The release mechanism changed with formulation. HPMC content was the predominant controlling factor. As the HPMC content increased, drug release rate decreased, and the release mechanism gradually changed from Higuchi diffusion release to case II transport. Additives increased the release rate and enhanced Fickian diffusion. As drug content increased, release rate calculated from percent release data decreased while that calculated from mg release data increased. When indomethacin content was lower, drug release was diffusion controlled and when higher, non-Fickian transport or case II transport was apparent.

Additive effects were also examined. Starch was found to most effectively maintain case II release. Complex additives containing starch were superior to any additive by itself.

A multiple regression model was used to determine the relationship between response (release rate) and factors (content of HPMC and diluents), and on the basis of this model a formulation was established and found valid by agreement with data from the regression model.
\end{abstract}

Key words hydroxypropylmethylcellulose; indomethacin; sustained release; regression analysis

The hydrophilic cellulose derivatives, hydroxypropylmethylcellulose (HPMC), have been widely used to control drug release from solid doses, such as from a sustained release or controlled release system. The mechanism for drug release is as follows ${ }^{11}$ : a) Water dissolves the drug at the surface, causing it to immediately release. Water penetrates the matrix via pores and brings about a gelling of the polymer. b) Water continuously penetrates the matrix, the gel expands and the drug is released. This is the main stage of the drug release process. c) When water has reached the center of the tablet and drug concentration decreases to less than its solubility, the drug release rate begins to fall. This is the tailing-off period.

Drug release rate can be adjusted by additives. The effect of additives on release is expansion of the gel barrier or prevention of continuous gel formation. ${ }^{1)}$

Though the above process is quite complicated, the drug release data can be explained by several equations, such as those proposed by Higuchi ${ }^{2)}$ and Higuchi. ${ }^{3)}$ In some cases, however, drug release has failed to follow such equations. ${ }^{4)}$

Little attention has been directed to the effects of the content of each component in a formulation on a mechanism; those of commonly used additives such as cornstarch and microcrystallinecellulose (MCC) on drug release in particular have not been reported. The object of the present study was to clarify the effect of the amount of water insoluble drug indomethacin (IND) and the amount and types of diluent on drug release rate or on drug release kinetics. A hydrophilic gel matrix was prepared and dissolution tests were carried out.

\section{Experimental}

Materials Metolose 90SH 4000SR (HPMC) (Shin-Etsu Chemical Co., Japan) was used as a hydrophilic polymer. IND (lot No. F-276,

* To whom correspondence should be addressed.
Sumitomo Pharmaceuticals Co., Japan), particle size $\langle 150 \mu \mathrm{m}$ fraction $\rangle$ was a model drug. Lactose (Pharmatose 200M, DMV Co., Holland), cornstarch (XX16, Nihon Shokuhin Kako Co., Japan) and MCC (Avicel pH 101, Asahi Kasei, Co., Japan) served as diluents.

Preparation of Matrix Tablets Flat-faced tablets $8.5 \mathrm{~mm}$ in diameter were prepared by directly compressing a sample powder at $1000 \mathrm{~kg} / \mathrm{cm}^{2}$ for $30 \mathrm{~s}$ under a hydraulic press (O. J. Shop, press model 10, Osaka Jack MFG. Co., Japan).

Formulations used for determining effects of additives are shown in Table I. Formulations $8-11$ with lactose replaced by starch, or with lactose replaced by $\mathrm{MCC}$ were also used to determine the effect of diluent

TABLE I. Formulations and $n$ for Matrix Tablets Containing IND and HPMC 2 Components and Containing IND, HPMC and Lactose 3 Components

\begin{tabular}{|c|c|c|c|c|}
\hline \multirow{2}{*}{$\begin{array}{c}\text { Formulation } \\
\text { No. }\end{array}$} & \multicolumn{3}{|c|}{ Formulation ammount (mg) } & \multirow{2}{*}{$n$} \\
\hline & IND & HPMC $90 \mathrm{SH} 4000$ & Lactose & \\
\hline 1 & 25 & 75 & - & 0.83 \\
\hline 2 & 50 & 75 & - & 0.85 \\
\hline 3 & 75 & 75 & - & 0.85 \\
\hline 4 & 125 & 75 & - & 0.84 \\
\hline 5 & 75 & 25 & - & 0.46 \\
\hline 6 & 75 & 50 & - & 0.92 \\
\hline 7 & 75 & 100 & - & 0.80 \\
\hline 8 & 25 & 50 & 75 & 0.26 \\
\hline 9 & 25 & 65 & 60 & 0.43 \\
\hline 10 & 25 & 75 & 50 & 0.83 \\
\hline 11 & 25 & 90 & 35 & 0.89 \\
\hline 12 & 50 & 25 & 75 & 0.29 \\
\hline 13 & 50 & 50 & 50 & 0.38 \\
\hline 14 & 50 & 60 & 40 & 0.43 \\
\hline 15 & 50 & 65 & 35 & 0.90 \\
\hline 16 & 60 & 15 & 75 & a) \\
\hline 17 & 60 & 40 & 50 & 0.30 \\
\hline 18 & 60 & 55 & 35 & 0.66 \\
\hline 19 & 75 & 40 & 35 & 0.39 \\
\hline 20 & 85 & 30 & 35 & 0.38 \\
\hline 21 & 100 & 15 & 35 & a) \\
\hline
\end{tabular}

a) Denoted that samples $(W \leqq 60 \%)$ were too few to calculate $n$.

(C) 1995 Pharmaceutical Society of Japan 
types.

Dissolution Studies Dissolution was measured by a dissolution tester (Toyama Sangyo Co., Japan). The tablets were put in a small basket identified here as JP XII, and the paddle method was used. Nine hundred $\mathrm{ml}$ of buffer solution, $\mathrm{pH} 7.2$ maintained at $37^{\circ} \mathrm{C}$ was used as dissolution medium. The stirring rate was $100 \mathrm{rpm}$. IND concentration was monitored with a spectrophotometer (UV-160, Shimadzu Co., Japan) at $318 \mathrm{~nm}$. Three replications were made for each batch of tablets.

Release Profile Analysis For theoretical analysis, the following mathematical models were used: 1) zero-order kinetics (Eq. 1) ${ }^{5}$; 2) first-order kinetics (Eq. 2) ${ }^{5}$; 3) Hixon Crowell's cube-root equation (Eq. $3)^{5)}$, and 4) Higuchi's square root of time equation (Eq. 4). ${ }^{2,3)}$ Equations are as follows:

$$
\begin{aligned}
& W=K_{1} t \\
& \ln (100-W)=\ln 100-K_{2} t \\
& (100-W)^{1 / 3}=100^{1 / 3}-K_{3} t \\
& W=K_{4} t^{1 / 2}
\end{aligned}
$$

TABLE II. Diffusional Exponent and Mechanism of Diffusional Release from Swellable Controlled Release System

\begin{tabular}{cl}
\hline \hline Diffusional exponent $n$ & \multicolumn{1}{c}{ Drug release mechanism } \\
\hline 0.45 & Fickian diffusion (case I diffusion) \\
$0.45 \leqq 0.89$ & Anomalous (non-Fickian) transport \\
0.89 & Case II transport \\
$>0.89$ & Super case II transport \\
\hline
\end{tabular}

$n=1$ means zero order release.

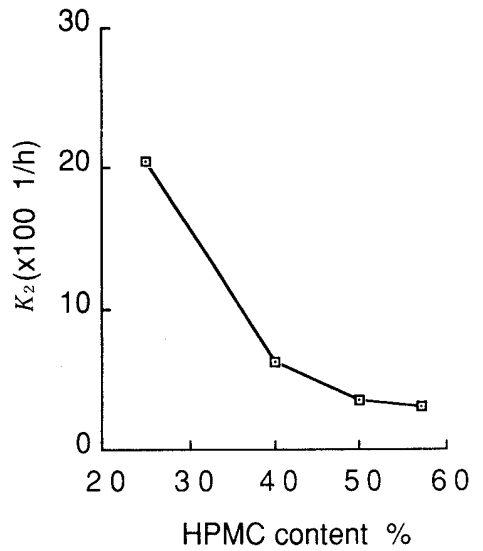

Fig. 1. Relationship between the Release Rate of IND and the Content of HPMC for Tablets Containing $75 \mathrm{mg}$ IND where $W$ is percent drug release at time $t$, and $K_{1}-K_{4}$ are release rate constants. During the research, we found that the release kinetics were affected by the content of polymer and diluents. Therefore, in this case, Eq. 5 was used to characterize the drug release profiles,

$$
W=K t^{n}
$$

where $n$ is the diffusional exponent and $K$, a constant incorporating the structural and geometric characteristics of the tablet. $n$ and the release mechanism for cylindrical tablets are shown in Table II. ${ }^{6,7)}$

\section{Results and Discussion}

Release Rate Analysis a) Release from the HPMCDrug 2 Component System: With increase in HPMC content, drug release rate decreased in matrices containing $75 \mathrm{mg}$ IND (Fig. 1). This could be explained by the swelling of HPMC after it absorbed water: a higher amount of HPMC absorbed more water and caused a greater degree of swelling. This, in turn, increased the tortuosity and the length of the drug diffusional path, decreasing the amount of drug released. ${ }^{8)}$

For tablets containing $75 \mathrm{mg}$ HPMC, IND release rate (calculated from percentage release data) decreased with the increase in dose (Fig. 2). This agrees with Mitchell et al., ${ }^{9)}$ who considered the presence of a drug to facilitate

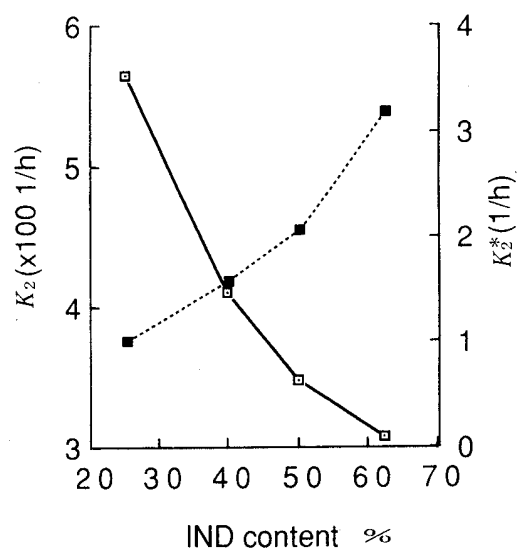

Fig. 2. The Relationship between Release Rate and IND Content for Tablets Containing $75 \mathrm{mg}$ HPMC

Dashed line, $K_{2}^{*}$ (calculated from $\mathrm{mg}$ release); solid line, $K_{2}$ (calculated from percent release).
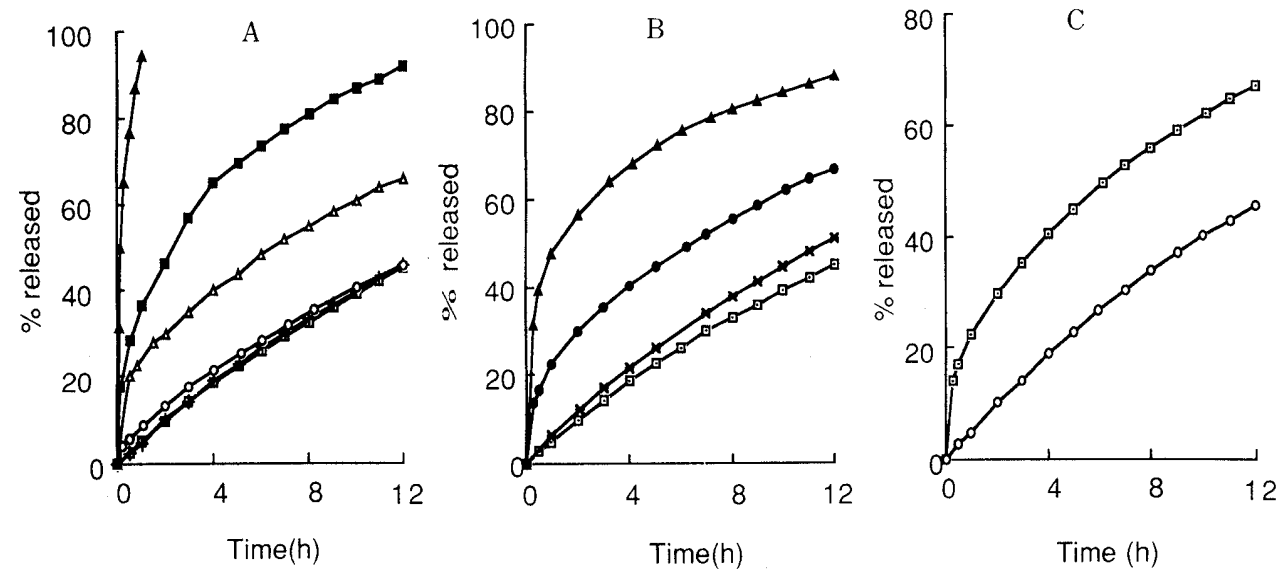

Fig. 3. Release of IND from Various Systems

A. Lactose (Lac) content constant system: $\square$, IND 25 Lac 35 HPMC 90 (mg); +, IND 50 Lac 35 HPMC 65 (mg); O, IND 60 Lac 35 HPMC 55 (mg); $\triangle$, IND 75 Lac 35 HPMC 40 (mg); $\mathbf{\square}$, IND 85 Lac 35 HPMC 30 (mg); $\mathbf{\Lambda}$, IND 100 Lac 35 HPMC 15 (mg).

B. IND content constant system: A, IND 25 Lac 75 HPMC 50 (mg); ๑. IND 25 Lac 60 HPMC 65 (mg); ×, IND 25 Lac 50 HPMC 75 (mg); ๑, IND 25 Lac 35 HPMC $90(\mathrm{mg})$.

C. HPMC content constant system: ๑, IND 25 Lac 60 HPMC 65 (mg); O, IND 50 Lac 35 HPMC 65 (mg). 
maintenance of the gel structure. But the release rate (calculated from weight release data) increased with the increase in dose. This demonstrated that, at a certain time $t$, the drug amount in dissolution medium also increased with the increase in dose.

b) Release of Drug From HPMC--Drug-Additive 3 Component System: Additives are often needed to maintain tablet size or to replace a portion of HPMC to modify drug release. Drug release characteristics from matrices containing additives were thus examined. The dissolution profiles are shown in Fig. 3.

Because the total tablet weight was kept at $150 \mathrm{mg}$, if the content of one of the 3 components was constant, then the contents of the others were altered. Drug release rate showed the following tendency: a) When the lactose content was kept constant, drug release rate decreased with the decrease in IND content, at IND/HPMC $\leqq 1$, release rate became constant (Fig. 4). b) When the IND content was kept constant, release rate increased with the decrease in HPMC content (Fig. 5). Therefore, it should be possible to modulate drug release rate even though HPMC is partially replaced by lactose. c) When the HPMC content was kept constant, release rate decreased with increased IND content (Fig. 6). This was consistent with the result of matrices without lactose.

Release Mechanism Analysis Based on the above kinetic models, the best linear relation was shown to be a first order kinetic model. But when HPMC content was greater, a very straight line was also observed for a zero order equation. At lower content, the best linear relation also fitted Higuchi's square root time equation, thus indicating the release mechanism to be changeable.

Empirical equation 5 was used to determine whether the release mechanism is affected by formulation change.

$n$ data for HPMC-IND 2 component matrices are listed in Table I. For tablets containing $75 \mathrm{mg} \mathrm{HPMC}$, this parameter was relatively constant at any IND content from $25 \%$ to $62.5 \%$. For tablets containing $75 \mathrm{mg}$ IND and varying amounts of HPMC, with HPMC content exceeding $40 \%, n$ showed little variation $(0.8-0.92)$, i.e. anomalous transport or case II transport. $n$ was 0.46 for a tablet containing 25\% HPMC; drug release from this tablet follows the Fickian diffusion model. The mechanism for drug release from an HPMC matrix thus appears to be controlled by HPMC content.

$n$ for the HPMC-IND-lactose 3 component system was not constant, and the following conclusions were drawn for a 3 component tablet with constant weight: 1) at the same IND content, as HPMC increased, $n$ increased; 2) at the same lactose content, $n$ increased with HPMC; 3 ) at the same HPMC content, $n$ increased with IND.

Variation in $n$ is shown in Figs. 46.

Though HPMC and IND enhanced $n$, the slopes of plots in these three figures indicated that $n$ was primarily determined by HPMC content. At HPMC $\geqq 50 \%$ for a 3 component tablet containing $25 \mathrm{mg}$ IND, the release mechanism was anomalous transport or case II transport. This agreed with the results of the HPMC and IND 2 component matrices. As HPMC content decreased, $n$ decreased. At $n=0.45$, Fickian diffusion was the predominant mechanism of drug release. In Table I, the

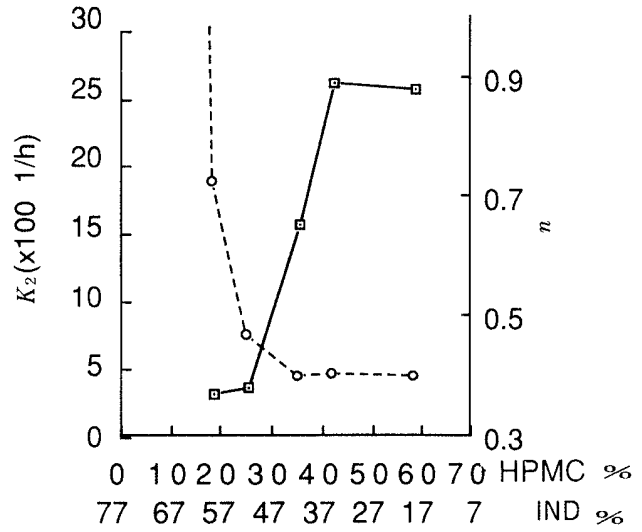

Fig. 4. IND Release Rate and $n$ as a Function of HPMC or IND Content (Lactose Content 23.33\%)

Dashed line, release rate $K_{2}$; solid, diffusional exponent $n$.

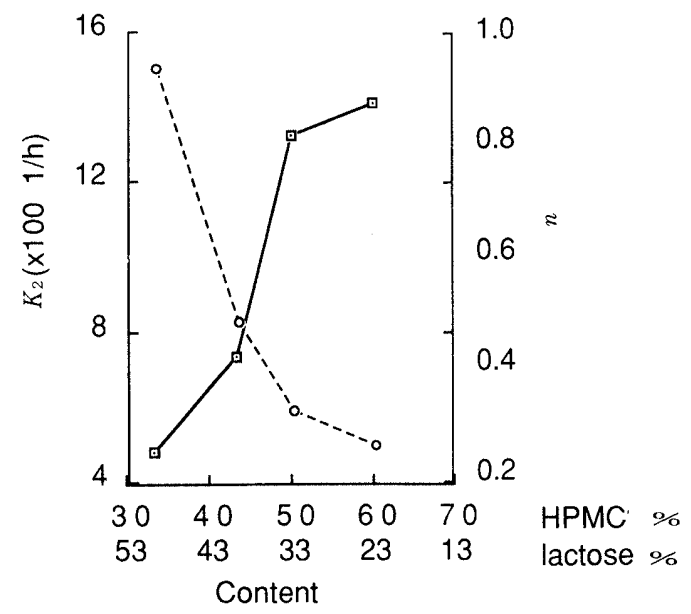

Fig. 5. IND Release Rate and Exponent $n$ as a Function of the Content of HPMC or Lactose (IND Content 16.7\%)

Dashed line, release rate $K_{2}$; solid line, diffusional exponent $n$.

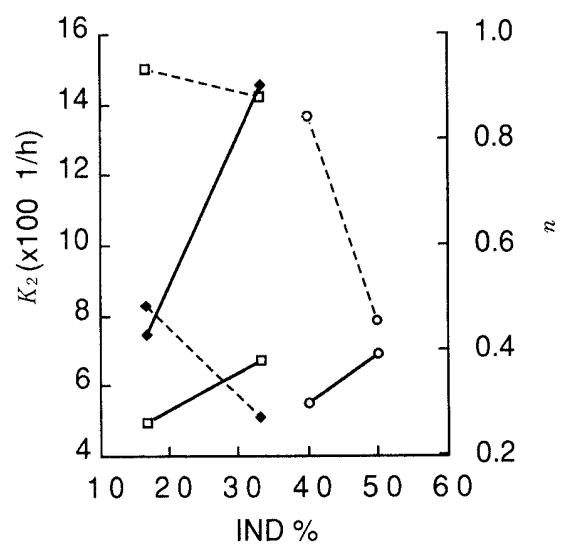

Fig. 6. IND Release Rate and Exponent $n$ as a Function of the Content of IND

Dashed line, release rate $K_{2}$; solid line, diffusional exponent $n$. HPMC content: O, $26.67 \% ; \square, 33.33 \% ; \bullet, 43.33 \%$.

$n$ values of some matrices are less than 0.45 , this was related to the structure of gel barrier. If HPMC content was relatively lower or diluent content was relatively higher, the amount of water-filled pores was relatively increased and the tortuosity of pores was smaller. The combined mechanism (diffusion partially through swollen 


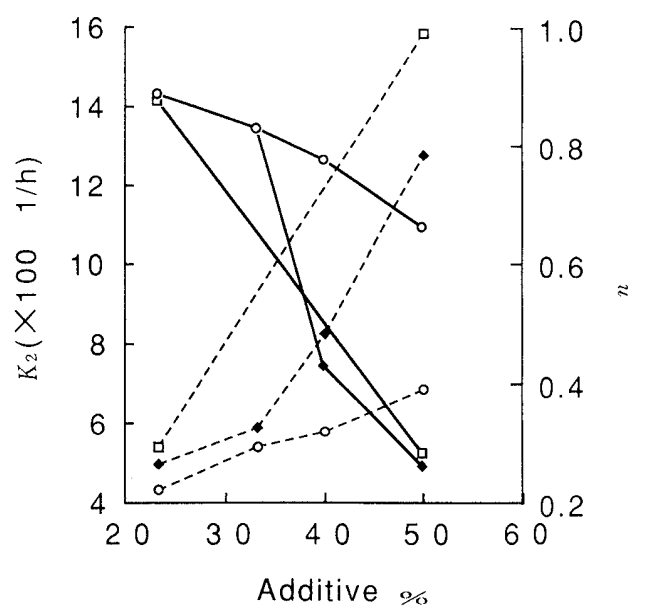

Fig. 7. IND Release Rate and Exponent $n$ as a Function of the Amount of the Additives

Dashed line. release rate $K_{2}$; solid line, diffusional exponent $n . \square$, MCC; lactose; $\bigcirc$, corn starch.

matrices and partially through water-filled pores) shifted the value of $n$ toward smaller values. ${ }^{10)}$ Therefore at $n<0.45$, IND release profiles showed the root of time dependence, i.e. Fickian diffusion. As noted by Ritger and Peppas, ${ }^{6}$ case I (Fickian diffusion) drug release behavior in a swelling controlled system is unique in that it can be expressed as a single parameter, the diffusion coefficient. Higuchi $^{2)}$ and Higuchi ${ }^{3)}$ derived equations containing this coefficient to describe drug release.

The drug release mechanism from HPMC matrices can be adjusted by changing the formulation of the tablet. Ideal zero order release is possible with high HPMC content. HPMC partially replaced by adding diluents may cause Fickian diffusion or anomalous transport.

Effects of Diluent Types After replacing lactose with $\mathrm{MCC}$, release was essentially the same in all cases though solubility and molecular weight for MCC and lactose differed considerably. Due to the disintegration of $\mathrm{MCC}$, formulations in which it was used caused somewhat faster drug release.

With cornstarch as the diluent in place of lactose, release changed greatly. Release for formulations containing starch was slower than for those containing lactose. At higher additive content, the difference was even more apparent and the same was also noted for $n$. The effects of additives on release and $n$ are shown in Fig. 7 .

The above differences in release rate and $n$ may possibly have been due to the solubility of lactose and cornstarch and their effects on tortuosity, according to Mitchell et al. ${ }^{9)}$ As a water soluble diluent dissolves, it diffuses outward and decreases tortuosity. Thus, with the increase in water soluble diluent, the diffusion mechanism became more important; the water insoluble diluent did not diffuse outward, but became entrapped in the matrix. Consequently, $n$ decreased very slowly.

Regression Analysis The above results showed starch to be the best additive. To investigate the contribution of HPMC and starch content to the release rate $K_{2}$, lactose or MCC was added to keep tablet weight at $150 \mathrm{mg}$. The effects of controlled variables on response variables were determined by the following model:

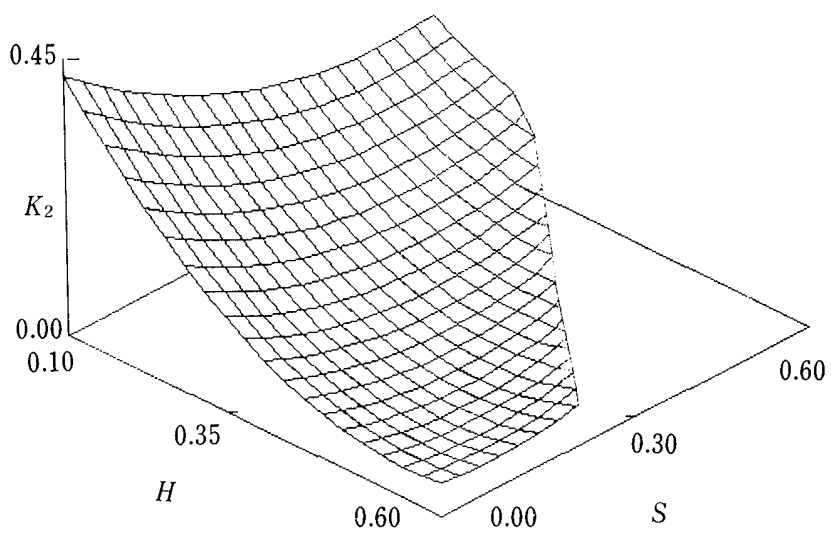

Fig. 8. The Effect of HPMC $(H)$ and Cornstarch (MCC) (S) Concentrations of Drug Release Rate $K_{2}$

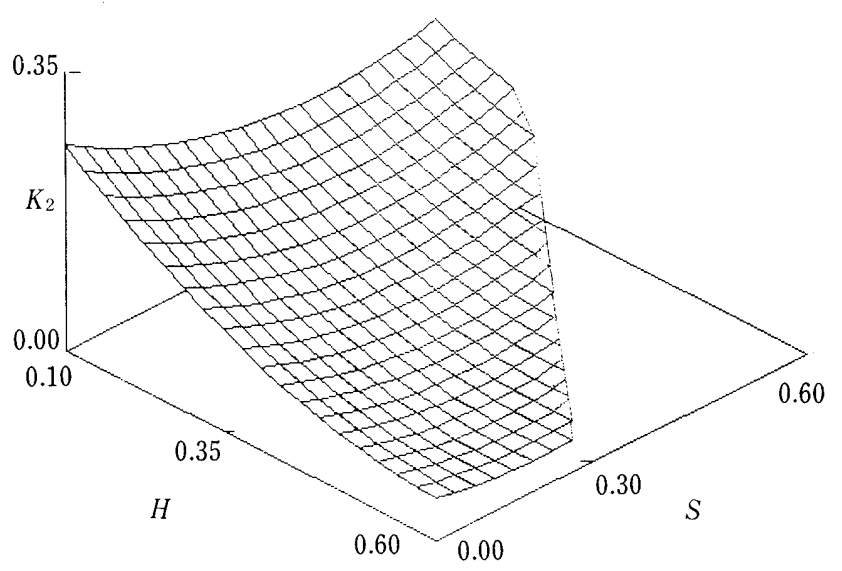

Fig. 9. The Effect of HPMC $(H)$ and Cornstarch $(S)$ Concentrations on Drug Release Rate $K_{2}$

$$
\begin{aligned}
& \text { response variable } K_{2} \\
& \quad=a+b_{1} * H+b_{2} * H^{2}+c_{1} * S+c_{2} * S_{2}+d * H * S
\end{aligned}
$$

where $K_{2}$ is a first order release rate constant, $H$ and $S$ are HPMC and starch content, respectively, $a$ is a constant, and $b_{1}, b_{2}, c_{1}, c_{2}$ and $d$ are coefficients. The regression equations obtained are:

$$
\begin{aligned}
& K_{2}=0.604-2.010 H+1.819 H^{2}-0.981 S+0.814 S^{2}+1.426 H * S \\
& N=17 \quad R^{2}=0.7827 \quad T(11)<0.05 \quad F(6,11)=16.62
\end{aligned}
$$

for matrices containing starch-MCC as complex additive and

$$
\begin{aligned}
& K_{2}=0.344-0.927 H+0.736 H^{2}-0.535 S+0.586 S^{2}+0.577 H * S \\
& N=18 \quad R^{2}=0.7694 \quad T(12)<0.05 \quad F(5,12)=16.22
\end{aligned}
$$

for matrices containing starch-lactose as complex additives, where $N$ is number of formulations, $R^{2}$, the correlation coefficient, $T$, the value of Student's $t$-test, and $F$, that obtained by the $F$-test. Based on these data, response surfaces were obtained (Figs. 8 and 9).

High HPMC concentrations usually retarded drug release significantly. The effects of complex additives, starch-lactose and starch-MCC, were unusual. Formulations with complex additives brought about relatively small release rates compared with those with single additives, thus, there was an optimal ratio of starch-lactose or starch-MCC.

Drug release from matrices containing these additives 
TABLE III. The Formulations and the Predicted and the Experiment Results

\begin{tabular}{|c|c|c|c|c|c|c|c|}
\hline \multirow{2}{*}{ No. } & \multicolumn{5}{|c|}{ Formulation $(\%)$} & \multirow{2}{*}{$\begin{array}{c}\text { Predicted } \\
K_{2}\end{array}$} & \multirow{2}{*}{$\begin{array}{l}\text { Experiment } \\
\qquad K_{2}\end{array}$} \\
\hline & IND & HPMC & Starch & $\mathrm{MCC}$ & Lactose & & \\
\hline 1 & 16.67 & 15.00 & 50.00 & 18.33 & - & 0.16 & 0.17 \\
\hline 2 & 16.67 & 15.00 & 40.00 & - & 28.33 & 0.14 & 0.18 \\
\hline
\end{tabular}

showed essentially the same mode of dissolution, but the release rate of tablets containing MCC was nearly twice that of those containing lactose, thus showing lactose to be the better additive of the two. Formulations were made based on the above results and were expected to release $80-100 \%$ of a drug within $12 \mathrm{~h}$. Experimental and calculated values are indicated in Table III and show agreement.

\section{References}

1) M. J. Vazquez, B. Perez-Marcos, J. L. Gomez-Amoza, R. Martinez-Pacheco, C. Souto, A. Concheiro, Drug Dev. Ind. Pharm., 18, 1355 (1992).

2) T. Higuchi, J. Pharm. Sci., 52, 1145 (1963).

3) W. I. Higuchi, J. Pharm. Sci., 51, 802 (1962).

4) E. Touitou, M. Donbrow, Int. J. Pharm., 11, 131 (1982).

5) A. K. Singla, D. K. Mediratta, Drug Dev. Ind. Pharm., 14, 1883 (1988).

6) P. L. Ritger, N. A. Peppas, J. Controlled Release, 5, 37 (1987).

7) J. W. Skong, M. V. Mikelsons, O. N. Vigneron, N. L. Stemm, J. Controlled Release, 27, 227 (1993).

8) L. S. C. Wan, P. W. S. Heng, L. F. Wong, Drug Dev. Ind. Pharm., 19, 1201 (1993).

9) K. Mitchell, J. L. Ford, D. J. Armstrong, P. N. C. Elliot, C. Rostron, J. E. Hogan, Int. J. Pharm., 100, 155 (1993).

10) N. A. Peppas, Pharm. Acta Helv., 60, 110 (1985). 